\title{
A fast and reliable approach to simulating the output from an X-ray tube used for developing security backscatter imaging.
}

\author{
A.Vella $^{* a}$, A.Munoz ${ }^{\mathrm{a}}$, M.J.F.Healy ${ }^{\mathrm{a}}$, D.W.Lane ${ }^{\mathrm{a}}$, D.Lockley ${ }^{\mathrm{b}}$, J.Zhou ${ }^{\mathrm{c}}$ \\ ${ }^{\mathrm{a}}$ Cranfield University, Shrivenham Campus, Watchfield, SN6 8LA, United Kingdom; ${ }^{\mathrm{b}}$ Counter \\ Terrorism \& Security Division, Dstl, Fort Halstead, United Kingdom; ${ }^{\mathrm{C}}$ VJ Technologies, 89 \\ Carlough Rd, Bohemia, NY 11716, USA
}

\begin{abstract}
The PENELOPE Monte Carlo simulation code was used alongside the SpekCalc code to simulate X-ray energy spectra from a VJ Technologies' X-ray generator at a range of anode voltages. The PENELOPE code is often utilised in medicine but is here applied to develop coded aperture and pinhole imaging systems for security purposes. The greater computational burden of PENELOPE over SpekCalc is warranted by its greater flexibility and output information. The model was designed using the PENGEOM sub-tool and consists of a tungsten anode and five layers of window materials. The photons generated by a mono-energetic electron beam are collected by a virtual detector placed after the last window layer, and this records the spatial, angular and energy distributions which are then used as the X-ray source for subsequent simulations. The process of storing X-ray outputs and using them as a virtual photon source can then be used efficiently for exploring a range of imaging conditions as the computationally expensive electron interactions in the anode need not be repeated. The modelled spectra were validated with experimentally determined spectra collected with an Amptek X-123 Cadmium Telluride detector placed in front of the source.
\end{abstract}

Keywords: Monte Carlo simulation, X-ray generator, X-ray spectrometry, CdTe detector, X-ray spectra

\section{INTRODUCTION}

Applications using X-ray imaging techniques have been widely used in industry, medicine and defence [1]. Knowledge of the energy spectrum produced by an X-ray tube is valuable, particularly for identifying different materials present. The aim of this work was to determine via Monte Carlo modelling the X-ray spectrum of VJ Technologies X-ray source. These spectra constitute the basis of the backscatter coded aperture imaging currently under development at Cranfield University. The X-ray source was simulated in PENELOPE [2] and SpekCalc [3] and the results validated by both measuring the spectra with an X-123 Amptek Cadmium Tellurite (CdTe) [4]. By simulating the real VJ technology Xray source separately from the coded aperture and test objects it is possible to increase computational speed and flexibility. By determining the source spectra separately for each voltage, and then storing its flux and energy distribution, the same X-ray source data can be input rapidly into a range of coded aperture, pinhole or object conditions without the need to simulate the same electron interactions into the target anode each time. Modelling the source is feasible to test conditions that may not be possible now, but may be possible in the future. Examples include operating the physical VJ tube at higher voltages than currently developed or if the anode was replaced with a different material other than tungsten. Of course, modelling even more theoretical sources is possible, such as monochromatic sources and potentially applying them to test what theoretical energy distributions best reveal certain features. Output energy spectra at a range of voltages were determined, which was information not available from the manufacturer. The modelled spectra were then compared to experimentally determined spectra collected with the X-123 Amptek CdTe detector placed in front of the VJ source.

${ }^{*}$ Corresponding author.

E-mail address: a.vella@cranfield.ac.uk. 


\section{MATERIALS AND METHODS}

\subsection{Modelling the VJ Technology $X$-ray source in PENELOPE}

PENELOPE [2] is a general Monte Carlo code that simulates coupled electron-photon transport by using a combination of numerical and analytical physical models to describe the interactions of electrons and photons with matter [5] [6]. Characteristic X-ray emission from targets irradiated by electron beams is usually quantified by means of the X-ray yield, which is defined as the average number of characteristic X-rays emerging from the target surface per unit solid angle and per incident electron [5].

The current real VJ tube setup comprises a tungsten target in vacuum behind five window layers. To model the tube, PENELOPE generates mono-energetic electrons and pursues their tracks into the target, and then the generated X-ray photons as they pass through layers of beryllium $(0.8 \mathrm{~mm}$ thickness $)$, glass $(1.5 \mathrm{~mm}$ thickness $)$, transformer oil (3mm thickness) and Ultem 1000 (1.5 mm thickness) (see figure 1). The model was designed in PENGEOM [7] and it consisted of a tungsten cylinder cut at 35 degrees on the right edge (in purple on figure 1), and four disks in the four different materials and thicknesses mentioned previously. The X-rays are produced when the electrons from the monoenergetic beam hits the tungsten target and then the photons irradiate the layers at 90 degrees in vacuum. The X-ray spectrum is continuous and emerges as a 40 degrees' cone from the multilayer window. To collect the data, a virtual detector was placed outside the window, to record the flux for use as the X-ray source for subsequent simulations, as described earlier. The virtual detector was set an energy bin width of $0.16 \mathrm{keV}$. Spectra were collected for anode voltages from $60 \mathrm{kV}$ up to $150 \mathrm{kV}$ in $10 \mathrm{kV}$ step where the number of simulated showers for each simulation was kept to $10^{7}$, which gives reasonable statistics in a relatively short time.

PENELOPE also allows the use of variance-reduction techniques to increase the efficiency of the simulation of specific low-probability events and it incorporates a subroutine package which automatically tracks particles through material systems consisting of homogeneous bodies limited by quadric surfaces. At the detector, the variance reduction technique was enabled in the input file to increase the accuracy of the spectrum [8].

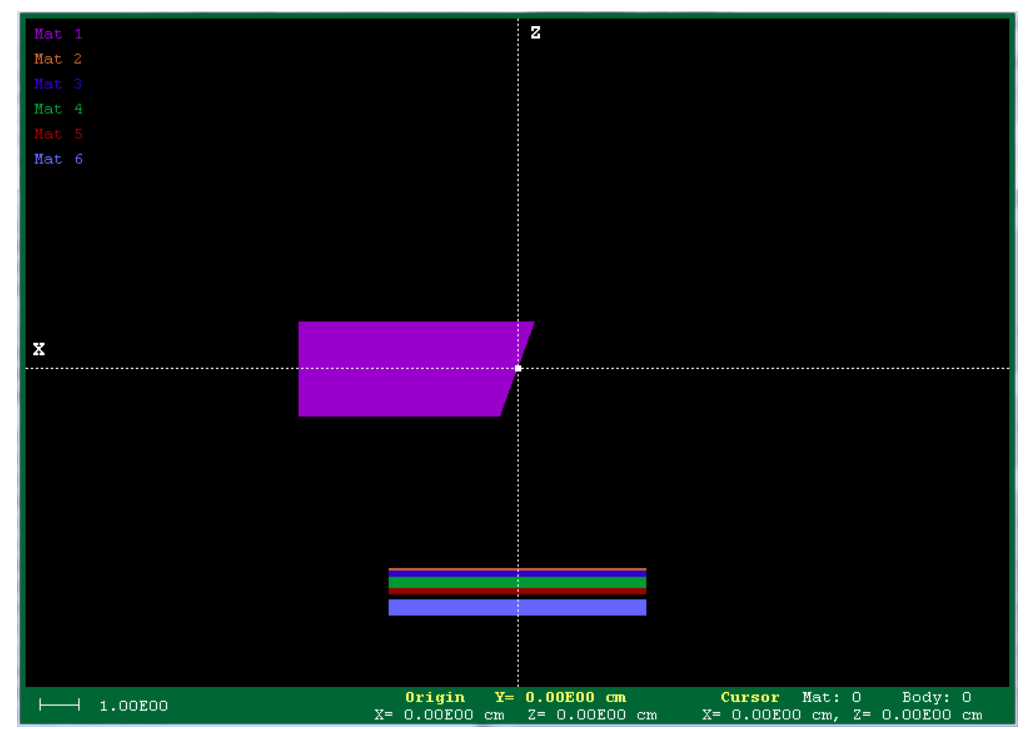

Figure 1. The model of the X-ray tube in PENELOPE. The model has been designed through PENGEOM sub-tool and consists of a tungsten target, the anode, and five layers of window material in vacuum. A mono-energetic beam of electrons hits the tungsten and then the photons irradiate the window at 90 degrees before being collected by a virtual detector.

\subsection{Simulation with SpekCalc}

To increase confidence in the output of the PENELOPE simulation, a comparison was made with SpekCalc [3]. This software creates a simple model including target, anode voltage and filters of different thickness. The theoretical approach underlying SpekCalc combines semi-empirical models of X-ray production, treating the penetration and energy 
loss of the incident electron beam crudely but quickly. It uses the pre-calculated survival probabilities for an electron reaching certain depths within the target, and, the electron energy distributions at those depths. SpekCalc allows the user to calculate, display and save, in energy bins of user-defined width, X-ray spectra emitted from tungsten anode tubes. The user selects the electron energy in $\mathrm{keV}$, the X-ray take-off angle and the amount of filtration by layer thicknesses. Filtration can be selected in $\mathrm{mm}$, for 7 materials: $\mathrm{Al}, \mathrm{Cu}, \mathrm{W}, \mathrm{Sn}, \mathrm{Be}$, water and air. The range of potentials that can be modelled is wide $(40-300 \mathrm{kV})$ making the utility useful in both diagnostic imaging and radiotherapy. Spectra are generated within a few seconds computation time. Although this program seems user-friendly, it presents some limitations: it is only possible to model the generation of X-rays rather than the use of X-rays beyond the generator and its filters.

\subsection{Measuring the VJ Technology X-ray source spectra}

The modelled spectra were then compared to spectra experimentally determined with an Amptek X-123 Cadmium Telluride (CdTe) detector placed in front of the VJT source. The experimental arrangements are shown in figure 2A. The $\mathrm{X}-123 \mathrm{CdTe}$ is a compact X-Ray and low energy Gamma Ray detector with a Beryllium window mounted on a Peltier type thermo-electric cooler keeping components at $-30^{\circ} \mathrm{C}$. The components are enclosed in a vacuum tight, light tight package and operation is possible in less than one minute from power turn-on. The X-123CdTe includes the detector, preamplifier, digital pulse processor and MCA, and power supply, and it connects via USB to PC [3]. A highly linear energy calibration was determined with ${ }^{241} \mathrm{Am}$ and ${ }^{238} \mathrm{Pu}$ gamma sources.

The detector was covered with a $0.5 \mathrm{~mm}$ aperture $10 \mathrm{~cm}$ thick tungsten collimator to decrease the photon flux, and so to reduce dead time and spectrum artefacts such as pulse pile up and sum peaks. However, further measures to reduce flux were required. The geometry of the room did not allow the detector-source distance to be increased significantly and it became necessary to decrease the intensity of the X-rays coming from the source. On request the manufacturer (VJ Technology) reduced the minimum operational current from $2 \mathrm{~mA}$ down to $0.2 \mathrm{~mA}$ by a software patch. To help shield the detector from X-rays backscattered from the walls, tables and objects in the room, and confine the signal to that coming from the source, the detector was shielded by surrounding it with lead bricks, leaving the collimated area directed towards the source. A lead lid then covered the castle seen in figure $2 \mathrm{~B}$.

These combined measures reduced the dead time below 1\% in all instances, including the highest anode voltage of $150 \mathrm{kV}$. The spectra were then collected using the manufacturer software ADMCA, and a correction of escape peaks later applied to the spectra.
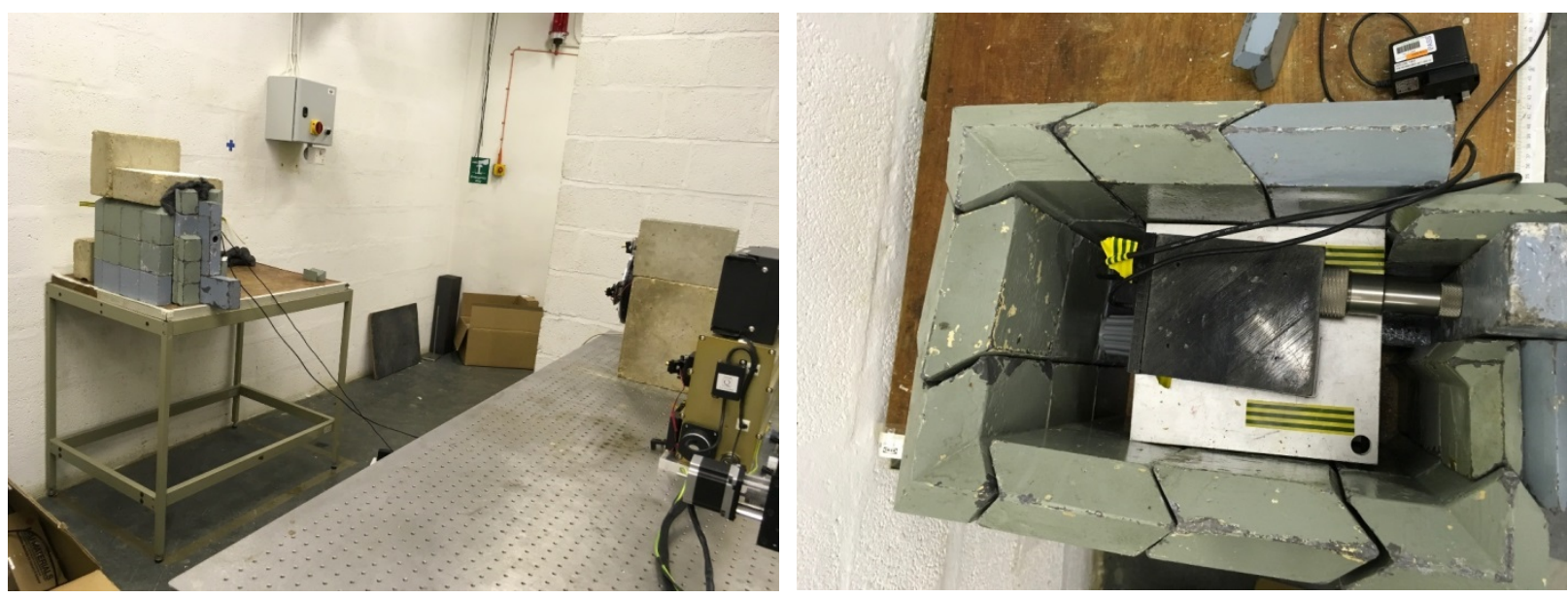

Figure 2A-B. Experimental setup for detecting the X-rays coming from the source with the CdTe detector. The detector was placed $1.50 \mathrm{~m}$ away from the source and shielded with lead bricks to minimise scatter from walls $(2 \mathrm{~A})$. It was aligned with the collimator by a support stage (2B). 


\section{RESULTS AND DISCUSSION}

\subsection{Energy spectra at different energies/voltages}

When comparing simulated with measured X-ray spectra, the following features should be considered. In PENELOPE, the results are expressed as energy-probability spectrum, while in SpekCalc and in the experiment the data were collected as energy-counts. Thus, normalisation was necessary to compare the results. The figures 3A-F show the comparisons between the PENELOPE simulated spectra (in red), the SpekCalc spectra (in blue) and the experiment (in green) at $70,90,100,120,130$, and $150 \mathrm{kV}$. The output data are normalized by defining intensity as:

$$
I(\text { a. u. })=\frac{\text { output on } Y \text { axis }}{\text { Maximum Bremsstrahlung(output on } Y \text { axis })}
$$

whereas the output on y axis is number of counts for the experimental and SpekCalc data, while in PENELOPE it is expressed in terms of probability $(1 \mathrm{leV})$. For the sake of simplicity, all the datasets were represented as continuous lines. The output results from simulations and experiment appear to be in good agreement, both in the bremsstrahlung area and the tungsten peaks. However, closer examination of the figures, reveal there are some systematic differences between the PENELOPE simulation and the experiment. At voltages up to $100 \mathrm{kV}$, it is possible to observe there is almost no difference between the simulations and the experiment. This difference was estimated as just $3 \%$ between the experimental and the PENELOPE data as shown in figure 4A. As the acceleration voltage of the tube rises, the fluorescence intensity of the tungsten peak increases in the experimental data marginally above that simulated by PENELOPE (figure 4B). This difference is possibly due to the presence in the experimental setup of a tungsten collimator, which generates more fluorescence in the experimental data than is simulated, as the tungsten collimator was not modelled. This means the experimental detector is not measuring purely the VJ X-ray generator but also some secondary fluorescence from the environment. It is also possible to note in the experimental spectra the presence of a low broad bump on the background spectrum at energies above those the tungsten fluorescence peaks at $150 \mathrm{kV}$. Some preliminary experiments including increased collimation suggest this bump arises from environmental scatter reaching the detector. It is also noticeable that at $150 \mathrm{kV}$ SpekCalc is overestimating the tungsten characteristic yield compared to experiment and PENELOPE. Possible reasons why SpekCalc overestimates is that whilst PENELOPE replicates the complex low $\mathrm{Z}$ multi layered window, SpekCalc can only simulate aluminium, tungsten and copper thus the attenuation will be different. Further, the default energy bin width of SpekCalc may in some cases misrepresent the X-ray yield. Where the $\mathrm{k} \alpha_{1}$ and $\mathrm{k} \alpha_{2}$ are not resolved, the combined peak height would exceed that from PENELOPE. If the yield was evaluated as the area under a line plot then the effect could be exacerbated by the large area formed as a high single value connects to points in adjacent bins.

Fundamentally, PENELOPE includes far more processes of absorption and re-radiation than SpekCalc so some difference is expected between the two simulations. However, within the current needs of modelling the VJ technology X-ray source for the purpose of security screening, the output of PENELOPE has been shown to be reliable and sufficiently consistent with other simulated and experimental data. Small differences may arise from environmental scattering, differences in the detail in which X-ray production is modelled in the two simulations and the criterion upon which the spectra were normalised against one another. The output from PENELOPE is intended to become the input for future predictive work on the scattering of a wide range of materials, structures and conditions which are all ill-defined, and hence the relatively small uncertainties demonstrated here will not be the dominant errors in our near-term application.

\section{CONCLUSIONS}

PENELOPE was used to model X-ray spectra from the real VJ Technology X-ray tube. The VJ source was modelled for anode voltages up to $150 \mathrm{kV}$. A successful validation of PENELOPE was achieved against the SpekCalc simulation software. To support the validation, an experiment with a CdTe detector placed in front of the source was conducted. Being able to model the correct X-ray spectrum output by the VJ source is a foundation for replicating the experimental backscatter imaging capability as realistically as possible, especially with a view to materials discrimination. 

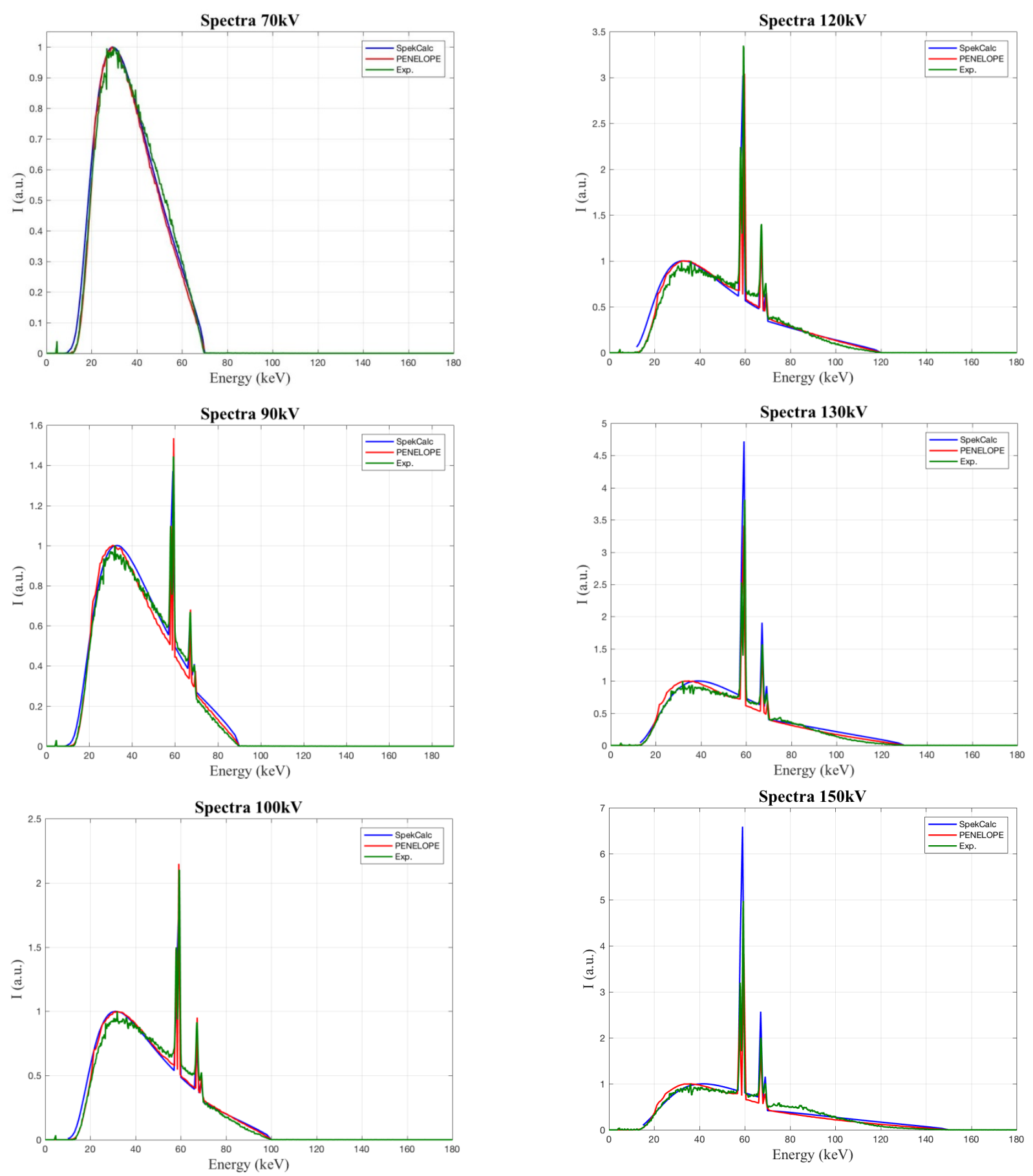

Figures 3A-B-C-D-E-F. X-ray spectra at 70 (3A), 90 (3B), 100 (3C), 120 (3D), 130 (3E) and $150 \mathrm{kV}(3 \mathrm{~F})$ simulated in PENELOPE (red), SpekCalc (blue) and experimentally measured with Amptek X123-CdTe detector (green). 

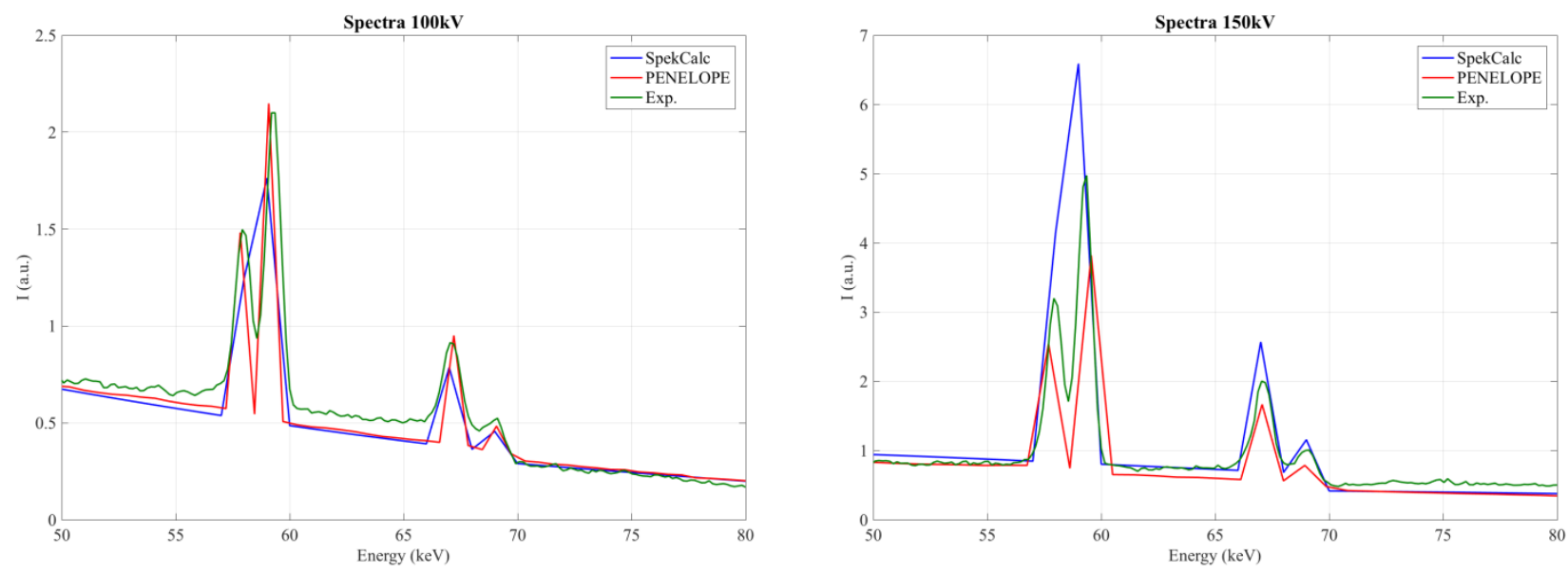

Figure 4A-B. Zoom of the X-ray spectrum at 100 (4A) and $150 \mathrm{kV}$ (4B) simulated in PENELOPE (red), SpekCalc (blue) and experimentally measured with Amptek X123-CdTe detector (green). The tungsten fluorescence peaks for the simulated and experimental data are well aligned at $100 \mathrm{kV}$ (just 3\% peak area difference between the experimental and PENELOPE data), while at higher energies $(150 \mathrm{kV})$ the experiment allows more fluorescence than the PENELOPE model, which underestimates the peak intensity with the respect to both the experimental and SpekCalc data.

\section{ACKNOWLEDGEMENT}

The authors would like to thank Dstl for the financial support for this work.

\section{References}

[1] M. Yücel, E. Emirhan, A. Emirhan, C. Emirhan and E. Barlas Yücel, "Comparison of simulated and measured spectra from an X-ray tube for the energies between 20 and $35 \mathrm{keV}$ " Nuclear Instruments and Methods in Physics Research A, vol. 799, pp. 50-53, 2015.

[2] F. Salvat, "PENELOPE-2014: A code system for Monte Carlo simulation of electron and photon transport," OECD/NEA Data Bank, Issy-les-Moulineaux, France, 2015.

[3] G. Poludniowski et al., "SpekCalc: a program to calculate photon spectra from tungsten anode X-ray tubes," no. 54, pp. 433-438, 2009.

[4] Amptek Material Analysis Division, “Amptek.com,” 2016. [Online]. Available: http://amptek.com/products/x-123cdte-complete-X-ray-gamma-ray-spectrometer-with-cdte-detector.

[5] D. Bote, X. Llovet and F. Salvat, "Monte Carlo simulation of characteristic X-ray emission from thick samples bombarded by kiloelectronvolt electrons," Journal of Physics D: Applied Physics, vol. 41, 2008.

[6] X. Llovet, L. Sorbier, C. S. Campos, E. Acosta and F. Salvat, "Monte Carlo simulation of X-ray spectra generted by kilo-electron-volt electrons," Journal of Applied Physics, vol. 93, no. 7, 2002.

[7] J. Almansa, F. Salvat-Pujol, G. Diaz-Londona, A. Carnicer, A. M. Lallena and F. Salvat, "PENGEOM - A general purpose geometry package for Monte Carlo simulation of radiation transport in complex material structures," Computer Physics Communications (in press), 2015.

[8] F. Salvat and J. M. Fernaddez-Varea, "Overview of physical interaction models for photon and electron transport used in Monte Carlo codes," vol. 46, pp. S122-S138, 2009.

[9] The MathWorks Inc., "MATLAB and Statistics Toolbox Release 2017a," 2014. [Online]. Available: https://uk.mathworks.com.

[10] F. Salvat, "Overview of physical interaction models for photon and electron transport used in Monte Carlo codes," Metrologia, vol. 46, no. 2, 2009. 
Content includes material subject to (C) Crown copyright (2017), Dstl. This material is licensed under the terms of the Open Government Licence except where otherwise stated. To view this licence,

visit http://www.nationalarchives.gov.uk/doc/open-government-licence/version/3 or write to the Information Policy Team, The National Archives, Kew, London TW9 4DU, or email:psi@,nationalarchives.gsi.gov.uk. 